\title{
Memory for modality of presentation: Within-modality discrimination*
}

\author{
LEAH L. LIGHT $\dagger$, CAROL STANSBURY, CHERYL RUBIN \\ Pitzer College, Claremont, California 91711
}

and

STAN LINDE

Claremont Men's College, Claremont, California 91711

\begin{abstract}
Two experiments demonstrated that Ss are capable of making within-modality memory discriminations in both visual and auditory modalities. In Experiment I Ss studied mixed lists of pictures and labels representing common objects and were subsequently required to judge whether the original presentation was pictorial or verbal. The high level of performance achieved on this task was unaffected by degree of categorical relatedness of items within method of presentation or by instructions to produce visual images when items were presented verbally. In Experiment II Ss demonstrated the ability to remember whether a sentence was originally presented by a male or a female speaker. Some strategies by which within-modality discrimination in memory might be accomplished are discussed.
\end{abstract}

In arguing against strength models of recognition memory, Anderson and Bower (1972) point out that "items which are equated on frequency and recency ... are nonetheless differentiable empirically on other grounds .... Example differentia in verbal learning experiments would be where in space the item was presented, who said it, how it was said, and other special characteristics of its physical and psychological context of presentation [p.99]." Underwood (1969) and Wickens (1970) have reviewed the literature dealing with several of these and other attributes of memory. Recently, experimentation in this area has been on the increase. For instance, Zechmeister and McKillip (1971) and Rothkopf (1971) reported that Ss are able to remember the spatial location of information in prose text. Similarly, several investigators have demonstrated that $\mathrm{Ss}$ in verbal learning experiments retain information about modality of presentation of items studied in mixed-modality lists; that is, Ss can identify words as having been presented either visually or auditorily (Bray \& Batchelder, 1972; Higby \& Light, unpublished study; Hintzman, Block, \& Inskeep, 1972). The purpose of the experiments reported here was to demonstrate that Ss can also make discriminations within sensory modalities in memory. In Experiment I Ss studied lists consisting of pictorial representations of some common objects and printed names of other objects and were subsequently required to indicate whether items were

*Experiment I was funded by grants to C. Stansbury from the Community Council and to L. L. Light from the Research and Development Committee of Pitzer College. Experiment II was carried out by C. Rubin and S. Linde. The authors thank Madeline Pinsky and Howard Fisher for preparing the materials used in Experiment I and Sara Schurr for assistance in data analysis. Dale Berger and Bill Banks made useful suggestions about statistical matters.

$\lceil$ Reprints should be requested from Leah L. Light, Department of Psychology, Pitzer College, Claremont, California 91711. studied as pictures or as labels. In Experiment II Ss listened to a tape recording of a number of sentences, half of which were spoken by a female $\mathrm{E}$ and half by a male $E$, and were then tested for retention of voice quality (sex of speaker). The experiments were thus intended to shed further light on S's ability to remember how something was presented and who said it.

\section{EXPERIMENT I}

In a pilot study, Ss studied a list of 40 items, 20 simple line drawings of familiar objects and 20 hand-printed labels for other familiar objects, and were then tested for memory of modality information either immediately or after a retention interval of 3 days. Twenty list items were associatively and categorically unrelated to each other, while the remaining 20 items were drawn from five taxonomic categories (four instances per category). Within each presentation condition (picture or label), half of the items were unrelated and the remaining half were taxonomically related. Further, two items from each taxonomic category were presented as labels and two as pictures for a given S. It may be argued that, if $S s$ rehearse categorically related items together during list input (see, e.g., Rundus, 1971), splitting categories over method of presentation might result in greater modality confusion for categorized than for unrelated items.

The main result of this pilot study-that Ss were able to remember whether an object was presented originally as a picture or as a label even after 3 days had elapsed-is quite dramatic. The overall mean proportions of correct responses given by Ss on the immediate and delayed tests were .95 and .83 , respectively. Also, ability to remember method of presentation was not much affected by degree of relatedness of items. Performance on unrelated items was not superior to performance on categorized items; for unrelated items, the mean 
proportion correct (collapsed over delay intervals and method of presentation) was .90 , while for categorized items this proportion was .89 .

Unfortunately, precise interpretation of the results of this pilot study is made difficult because the memory test used here required Ss to discriminate between two methods of presentation but did not require them to discriminate between old and new objects per se by including previously unpresented objects in the test and requiring an "old-new" judgment on the object itself, as well as a decision about method of presentation. Hence, it is not possible to determine whether the poorer performance on the delayed test should be attributed to inability to remember whether an item was presented at all or to inability to remember whether it was represented by a picture or a label. Also, it is impossible to assess effects involving the picture-label factor here, since no adequate measure of response bias is available.

Experiment I constituted a partial replication of the pilot study but employed a two-alternative forced-choice recognition test in addition to a within-modality discrimination (WMD) test, so that the effects of item loss (forgetting that a particular item was presented) could be separated from those of loss of modality information. Objects were presented either as pictures or as labels. However, photographic slides of real objects rather than line drawings were employed. The degree of relatedness factor was also manipulated. One-third of the to-be-remembered objects were judged to be taxonomically unrelated. The remaining two-thirds of the objects were instances of well-defined taxonomic categories. The items of half of these categories were split within modality, so that two items were presented as pictures and two as labels. For the remaining categories, all instances were presented in the same way; either all four category members were represented as pictures or all four category members appeared as labels for a given $S$. Retention interval was represented by a single level in Experiment I; all Ss were tested after a $10-$ min filled retention interval.

One additional variable was manipulated in Experiment I. Half of the Ss were instructed to form vivid mental images of objects whose labels wer? presented for study. Bower and Winchester (cited in Anderson \& Bower, 1972) have reported that, following a word association test, Ss are very accurate in discriminating E-presented stimulus words from their own associative responses. The present experiment explored the question of whether imagery instructions would affect Ss' ability to remember whether a previously studied object was presented as a label or as a picture. Phrased somewhat differently, this study investigated the question of whether Ss can discriminate between their own self-produced images and externally presented images.

\section{Method}

\section{Materials}

The to-be-remembered list consisted of slides representing 48 objects, 24 photographs and 24 typewritten labels. In a pilot study, $10 \mathrm{Ss}$ ranging in age from 19 to 42 years viewed 121 full-color photographic slides depicting common objects and were asked to assign simple one- or two-word labels to them. All photographs used in the to-be-remembered list in the main study were given the same label by at least 8 of these $10 \mathrm{Ss}$; such discrepancies as did occur in naming of to-be-remembered objects were minor-"slip" might be called "half slip" or "shoe" might be called "man's shoe." Of the 48 to-be-remembered items, 16 were unrelated [i.e., no two appeared as responses to the same category name in the Battig \& Montague (1969) norms], with 8 appearing as pictures and 8 as labels for a given $\mathrm{S}$. The remaining 32 items were four instances of each of eight taxonomic categories represented in the Battig and Montague norms. These eight categories were randomly assigned to two experimental conditions: same and split. All instances of a given category were presented either as pictures or as labels in the same condition; in the split condition, two of the four instances of a given category were randomly chosen to appear as pictures and two as labels. Categories assigned to the same condition were "a carpenter's tool, a type of vehicle, an article of clothing, a fruit;" those assigned to the split condition were "an article of furniture, a kitchen utensil, a part of the human body, a vegetable." Unrelated items included "basket, broom, map, flag." Labels were also presented as slides; words were typed in capitals on tracing paper and inserted in Kodak ready mounts.

The three degrees of relatedness of items (unrelated, same, split) and two methods of presentation (pictures, labels) generate six within-S experimental conditions. For the to-be-remembered list, items in these conditions were randomized in blocks of six, with no condition appearing more than once in each block of six and no two successive items being from the same condition. Two presentation lists were used equally often for Ss receiving imagery instructions and for $S$ s not receiving imagery instructions. Items appearing as pictures in one list appeared as labels in the other and vice versa.

Distractors for the two-alternative forced-choice recognition test were selected as follows. For the unrelated items, distractors were drawn from the pool of unrelated objects for which high between-S agreement on labels was achieved. Distractors for the categorized items belonged to the same Battig and Montague categories as did the to-be-remembered items with which they were paired; input items and distractors were roughly matched for taxonomic frequency and all were represented in the 25 most frequent responses to each category name. For the recognition test, a second random order of the to-be-remembered items was constructed, with the same restrictions as applied to the study order applying to the test order. Order of correct alternatives within test pairs was randomly determined.

\section{Subjects and Procedure}

All Ss for the main experiment were high school and college students recruited by means of an advertisement in a local newspaper. They were paid $\$ 2$ for their participation. The $S s$ were tested either individually or in groups of up to four Ss at a time, with all $S s$ in a given test session serving in the same imagery condition. Thirty-four Ss were tested. The protocol of one $S$ who failed to follow instructions was discarded. One additional protocol was randomly discarded from the imagery condition to equate the number of $S s$ in the two imagery instructions conditions; 16 Ss served in each imagery instructions condition.

The to-be-remembered list was presented by a Kodak Carousel 800 slide projector, set at a 5 -sec rate. All Ss were informed prior to list input that they would see a number of slides depicting common objects and that some of the slides would be readily identifiable photographs of everyday objects, while others would be the names of different objects. They were asked to attend carefully to what they saw and were told that a memory test would follow list presentation; the nature of the memory test was not divulged at this time. Those Ss serving in the imagery 
condition were further instructed that when the name of an object was presented they should form a vivid mental image of that object, as though it too were represented by a photograph; they were given the example of a "hot dog" that might be visualized "on a bun with mustard and pickle relish."

To avoid ceiling effects, $S$ s were engaged in an anagram solution task for $10 \mathrm{~min}$ following list presentation. The memory test was then administered. Each $S$ received a single sheet of paper with the numbers $1-48$, each followed by a short line and the words "picture" and "label." The $E$ then read the 48 test pairs aloud and $S$ indicated on his answer sheet whether the first or second item in each pair had somehow been represented on the input list by writing " 1 " or " 2 " on the blank line; $S$ then indicated whether the original representation was a picture or a label by circling the appropriate word. The recognition test was self-paced.

\section{Results}

Two measures were obtained for each S (see Table 1). First, the proportion of correct recognition choices was computed for each $\mathrm{S}$ for each of the six within-Ss cells. Second, within-modality discrimination (WMD) was conditionalized on correct recognition by considering modality choices only for those items that $S$ had correctly recognized. An arc sine transformation was applied to all proportions because the distributions of scores were skewed. The transformed scores for the two measures were submitted to separate three-way analyses of variance, with repeated measures on two factors (degree of relatedness and method of presentation).

The only effect to achieve significance when proportion of correct recognitions was considered was method of presentation $[F(1,30)=11.80, p<.01]$, with pictures being correctly recognized more often than labels. The picture-label difference was not in evidence when WMD was conditionalized on correct recognition $(\mathrm{F}<1)$. No other main effects or interactions reached significance at the .05 level in either analysis.

Inspection of Table 1 reveals that performance on the WMD task is excellent. Given the failure of any analysis to detect a difference between imagery conditions, mean proportion correct WMD scores were collapsed across imagery conditions. The mean conditional WMD proportion correct was 92 . Using a standard all-or-none guessing correction here gives a figure of .84 for the proportion of picture-label distinctions retained, given that a correct recognition choice was made. The hypothesis that the population proportion is .50 (or rather that the arc sine of the mean population proportion is 1.5708$)$ can be rejected at well beyond the .001 level of significance $[t(31)=24.16]$.

\section{Discussion}

The results of this experiment constitute a strong demonstration that $\mathrm{Ss}$ can make within-modality discriminations in memory when items are presented visually. In a similar vein, Kaplan, Kaplan, and Sampson (1968) and Sampson (1970) have reported studies in which Ss viewed mixed lists of drawings and labels and
Table 1

Experiment I: Proportion Correct Recognition and Conditional Proportion WMD

\begin{tabular}{|c|c|c|c|c|c|c|}
\hline & \multicolumn{3}{|c|}{ Pictures } & \multicolumn{3}{|c|}{ Labels } \\
\hline & $\begin{array}{l}\text { Unre- } \\
\text { lated }\end{array}$ & Same & Split & $\begin{array}{l}\text { Unre- } \\
\text { lated }\end{array}$ & Same & Split \\
\hline \multicolumn{7}{|c|}{ Proportion Correct Recognition } \\
\hline \multicolumn{7}{|c|}{ Imagery } \\
\hline $\mathbf{M}$ & .97 & .95 & .96 & .94 & .95 & .90 \\
\hline SD & .07 & .10 & .06 & .08 & .08 & .15 \\
\hline \multicolumn{7}{|c|}{ No Imagery } \\
\hline M & .95 & .96 & .94 & .90 & .87 & .93 \\
\hline $\mathrm{SD}$ & .09 & .06 & .13 & .11 & .14 & .10 \\
\hline \multicolumn{7}{|c|}{$\begin{array}{l}\text { Conditional Proportion WMD } \\
\text { Imagery }\end{array}$} \\
\hline $\mathbf{M}$ & .94 & .92 & .89 & .92 & .94 & .89 \\
\hline SD & .09 & .09 & .15 & .11 & .13 & .16 \\
\hline \multicolumn{7}{|c|}{ No Imagery } \\
\hline M & .95 & .93 & .93 & .90 & .95 & .88 \\
\hline SD & .13 & .07 & .10 & .17 & .09 & .17 \\
\hline
\end{tabular}

were subsequently tested by written recall and encouraged to reproduce what they had seen as accurately as possible. Under these conditions, Ss typically made very few reversal errors; that is, they rarely produced a drawing when a label was shown or a label when a picture was presented. Davies (1971) also discusses an unpublished investigation in which Ss who were exposed to mixed lists of labels, pictures, and pictures plus labels indicated how recalled items were input with a high degree of accuracy.

Within-modality discrimination appears to be unaffected by a number of procedural variables. First, neither the pilot study nor the main experiment revealed any effect of degree of item relatedness on WMD; WMD performance was not better on unrelated than on taxonomically related items. Either Ss did not utilize categorical relationships to guide choice of rehearsal strategy under the experimental conditions prevailing here (see Rundus, 1971) or rehearsal strategy does not affect maintenance of within-modality discrimination in memory.

Second, performance is probably not a function of whether the item is presented initially as a picture or as a label. Although recognition scores' were higher for items presented originally as pictures in Experiment I, no differences between WMD scores for pictures and labels could be detected when these scores were conditionalized on correct recognition. The latter finding also suggests that response bias was not a problem here; however, when picture-label choices for items on which $\mathrm{S}$ failed to choose the correct alternative on the recognition test were examined, a .68 response bias in favor of saying "label" was found. Thus, conclusions with regard to the picture-label factor must be tentative.

Third, ability to perform a visual WMD task does not appear to be dependent on the modality in which the 
Table 2

Experiment II: Proportion Correct Recognition and Conditional Proportion WMD

\begin{tabular}{|c|c|c|c|c|}
\hline & \multicolumn{2}{|c|}{$\begin{array}{l}\text { Proportion Correct } \\
\text { Recognition }\end{array}$} & \multicolumn{2}{|c|}{$\begin{array}{c}\text { Conditional } \\
\text { Proportion WMD }\end{array}$} \\
\hline & Male & Female & Male & Female \\
\hline \multicolumn{5}{|c|}{ Informed } \\
\hline M & .88 & .89 & .75 & .78 \\
\hline SD & .08 & .07 & .10 & .17 \\
\hline \multicolumn{5}{|c|}{ Uninformed } \\
\hline M & .86 & .87 & .67 & .62 \\
\hline SD & .13 & .12 & .20 & .16 \\
\hline
\end{tabular}

test is administered. In the pilot study, the memory test was given visually, whereas in Experiment 1 it was administered auditorily.

Fourth, ability to perform the WMD task persists over relatively long time intervals. In the pilot study, the memory test was given either immediately or after 3 days. In Experiment I WMD after a 10-min filled delay interval was found to be excellent.

Fifth, visual WMD was unaffected by instructions to form vivid mental images whenever the input representation of an object was a printed label. Thus, Ss are able to discriminate between self-produced images and experimentally presented pictures. In fact, although the data do not reveal any differential effects of imagery instructions, informal comments by Ss following debriefing about the purpose of Experiment I suggest that Ss believe that imagery instructions facilitate WMD. Several Ss reported that their own images were quite idiosyncratic and highly personal (e.g., "for 'shoe' I visualized my sandals") and, therefore, readily discriminable from remembered photographic images.

\section{EXPERIMENT II}

Experiment I demonstrated the existence of the WMD phenomenon for visual stimuli. The main purpose of Experiment II was to demonstrate WMD for auditory stimuli. The Ss in this study listened to a tape recording of 40 sentences, 20 spoken by a male $E$ and 20 by a female $E$, and were then given a combined two-alternative forced-choice recognition and WMD test.

A secondary purpose of this experiment was to investigate the effects of informing Ss prior to list presentation about the nature of the WMD task and instructing them to attend carefully to within-modality differences. Half of the Ss in this study were informed about the WMD test, while the remaining half were not informed. The question of interest is thus whether deliberate attention to within-modality differences in stimuli during initial presentation enhances performance on a subsequent WMD task.

\section{Method}

\section{Materials}

Eighty sentences, ranging in length from 7 to 13 words and dealing with current news events, were selected from a Sunday edition of the Los Angeles Times. Typical of the sentences selected are the following: "The crowd gave him the warmest ovation in memory; the understanding was that the entire match would be held in Paris; the style of a cart model is usually good for about 4 years; the new water supply is also expected to expand the mining operations." Of these sentences, 40 were assigned randomly to the presentation list and the remaining 40 served as distractors on the recognition test. The 40 presentation sentences were divided randomly into two groups of 20 sentences each, and the two sublists were recorded by one male and one female E. Sentence order was random with respect to sex of speaker. All sentences were spoken in a monotone, with approximately $2 \mathrm{sec}$ of silence separating successive sentences. Two versions of the presentation list were prepared, with sentences spoken by the female $E$ in one version recorded by the male $E$ in the second version and vice versa. The total length of each tape recording was about $5 \mathrm{~min}$. The two input lists were used equally often for the informed and uninformed Ss.

\section{Subjects and Procedure}

The Ss were undergraduates enrolled at the Claremont Colleges. Thirty-two Ss were tested but the protocols of two Ss who failed to follow instructions were discarded. To equate frequency of input lists, the protocols of one $S$ in the informed group and one $S$ in the uninformed group were discarded at random, leaving $14 \mathrm{Ss}$ in each of these two experimental conditions. The $\mathrm{Ss}$ were tested in groups of two to eight, with all Ss in a given session hearing the same input list and serving in the same instructions condition.

All Ss were informed that they would hear a tape recording of a number of sentences and that they should at tend carefully to sentence content, since a memory test would be forthcoming. Those Ss who served in the informed condition were further instructed that half the sentences were spoken by a male voice and half by a female voice and that they would be required to remember the voice in which a sentence had been spoken as well as the contents of the sentence.

Immediately following sentence presentation, Ss were given a written two-alternative forced-choice recognition and WMD test. Each input sentence was paired with a distractor sentence from the original sentence pool, with order of correct sentences within pairs being randomly determined. The 40 sentence pairs were presented in a 4-page test booklet. Following each sentence were the letters " $M$ " and "F." The S's task was to select the previously presented sentence in each pair by placing a checkmark in a space preceding it and to indicate sex of speaker by circling the appropriate letter. The test was self-paced.

\section{Results}

As in Experiment $I$, proportion correct recognition and conditional proportion WMD scores were computed for each S (see Table 2). An arc sine transformation was applied to these proportions and all statistical analyses were performed on the transformed scores. Response bias was not a problem in this experiment. The Ss did not show a strong preference for responding "male" or "female" on the WMD task. When those items for which $S$ made an incorrect recognition decision were considered, it was found that .51 of the voice judgments were "male" and .49 were "female."

A two-way analysis of variance with repeated measures on voice of speaker indicated no effects of either instructions or voice of speaker on proportion correct recognition, all Fs $<1$. A similar analysis of the conditional proportion WMD scores revealed only a 
marginally significant effect of instructions $[F(1,26)=4.71, p<.05]$, with the informed group performing slightly better than the uninformed group.

Performance on the auditory WMD task was not quite so impressive as on the visual WMD task in Experiment $\mathbf{I}$. The mean conditional proportion WMD scores, collapsed over voice of speaker, were .76 and .64 for the informed and uninformed groups, respectively. Applying an all or none guessing correction to these proportions indicates that the voice distinction was maintained for .52 of the sentences for the informed group and for .28 of the sentences for the uninformed group. The latter proportion is quite comparable to othe .18 figure obtained by Hintzman, Block, and Inskeep (1972) for voice judgments when Ss were unaware that they would be required to remember voice of speaker. The hypotheses that the population proportions for WMD are .50 may be rejected $[t(13)=8.03, p<.001$ for the informed group and $t(13)=2.99, p<.02$ for the uninformed group]. Thus, WMD may be found in the auditory as well as in the visual modality.

The conditional WMD results are difficult to interpret. Bray and Batchelder (1972) reported that Ss informed that they might later be required to remember in which of two modalities (auditory or visual) words had been presented did not perform differently than Ss who had no such advance information. Bray and Batchelder suggest that modality information is automatically entered into memory when Ss store studied items. The current results are not in conflict with those of Bray and Batchelder but suggest that prior instructions to attend to the relevant within-modality aspects of the input material may improve WMD. Given the very minimal level of significance of the difference between the two information conditions, however, this suggestion is offered with some reservations.

\section{DISCUSSION}

The experiments described here provide evidence that Ss can remember quite a lot about how an item was presented. Experiment I demonstrated that information about method of presentation within the visual modality persists over time, that WMD does not require identity of input and test modalities, that WMD is unaffected by degree of categorical relatedness among input items, and that imagery instructions do not affect WMD performance. Experiment II demonstrated the WMD phenomenon for stimuli presented in the auditory modality but was inconclusive with respect to the effects of advance knowledge about the WMD task on subsequent performance.

The present findings are in basic agreement with the results of Hintzman, Block, and Inskeep (1972), which became available in the literature while this manuscript was in preparation. These authors reported that both between- and within-modality distinctions were retained for a period of several minutes following list presentation. The within-modality discriminations investigated were block vs script letters in the visual modality and male vs female voice in the auditory modality. The .64 value for conditional proportion correct voice judgments for the uninformed group in Experiment II of the present series is entirely comparable to the .59 value of Hintzman et al. However, performance on the picture-label task in Experiment $I$ is clearly better (mean conditional proportion correct WMD $=.92$ ) than that reported for the Hintzman et al within-modality visual task (.58) or that reported for between-modality discrimination by either Hintzman et al (.74) or Bray and Batchelder (.80). Finally, Hintzman et al found that making spatial cues redundant with input modality did not affect voice judgments; this finding parallels the Experiment I result that degree of categorization had little if any impact on picture-label discrimination.

Our results, together with those of Bray and Batchelder (1972), suggest that storage of modality information may be an automatic process rather than a process that is under $S$ control, as hypothesized by Shiffrin and Atkinson (1969). The question that arises, then, is one of the utility of storing such information about sensory attributes of stimuli. Underwood (1969, p. 569) has argued that modality attributes probably function primarily as aids in event discrimination rather than as retrieval cues: Although Bray and Batchelder did not obtain clustering in free recall on the basis of different sensory modalities, Hintzman, Block, and Inskeep (1972) reported significant amounts of clustering both when words were varied between modalities and when they were varied within a single modality. Also, Murdock and Carey (1972) and Murdock and Walker (1969) have presented evidence that modality may provide a basis for organization in free recall. Thus, the bulk of the evidence currently available is consistent with the hypothesis that input modality can function as a basis for retrieval.

If modality information also serves a discriminative function, then one might predict that recognition memory should be best when modality attributes of list items. are held constant for study and test. However, the information available on this point is somewhat ambiguous. An experiment by Davies (1969) supports this hypothesis for pictures and labels, but Jenkins, Neale, and Deno (1967) found that recognition memory was better when items were both presented and tested as pictures than when either the presentation list or the test list or both consisted of labels. Neither Hintzman, Block and Inskeep (1972) nor Higby and Light (unpublished study) found a significant interaction between modality of input and modality of test when words were presented visually and auditorily. Hintzman et al did report an interaction between presentation and test modalities when type face of items was varied, but this effect was obtained only when items were presented in script. 
One mechanism that might underlie ability to perform visual WMD tasks is suggested by Paivio's $(1969,1971$, p. 179) dual-coding model. Paivio argues that items may be stored in either one or both of two memory codes-images and verbal labels. Pictures of common and easily nameable objects (such as those used in Experiment I) arouse both an image and a verbal label, but the imaginal code is more readily available. Concrete words (such as the labels of the objects used here) also arouse both codes but the verbal code is more readily available in this case. It is possible that, when a code is aroused, $S$ records not only the product of applying the code but also the fact that a particular code was employed. Such a mechanism would explain the absence of any effects of imagery instructions in Experiment I; S stores images for items represented on the input list as labels but also records what transformations on the input were needed to arrive at these images. When $S$ is later confronted by the WMD task, the original representation is recovered by examining the stored representation and the transformation récord. A similar process has been postulated by Prytulak (1971) to account for natural language mediation in a variety of tasks. An extension of this mechanism to WMD tasks requiring retention of voice quality, though perhaps somewhat artificial, might involve recording the nature of frequency analyses performed during speech perception.

\section{REFERENCES}

Anderson, J. R., \& Bower, G. H. Recognition and retrieval processes in free recall. Psychological Review, 1972, 79, 97-123.

Battig, W. F., \& Montague, W. E. Category norms for verbal items in 56 categories: A replication and extension of the Connecticut category norms. Journal of Experimental Psychology, 1969, 80 (3, Part 2).

Bray, N. W., \& Batchelder, W. H. Effects of instructions and retention interval on memory of presentation mode. Journal of Verbal Learning \& Verbal Behavior, 1972, 11, 367-374.

Davies, G. M. Recognition memory for pictured and named objects. Journal of Experimental Child Psychology, 1969, 7, 448-458.
Davies, G. M. The influence of verbal labelling on the retention of picture stimuli: An alternative viewpoint. Journal of Special Education, 1971, 5, 323-336.

Hintzman, D. L., Block, R. A., \& Inskeep, N. R. Memory for mode of input. Journal of Verbal Learning \& Verbal Behavior, 1972, 11, 741-749.

Jenkins, J. R., Neale, D. C., \& Deno, S. L. Differential memory for picture and word stimuli. Joumal of Educational Psychology, 1967, 58, 303-307.

Kaplan, S., Kaplan, R., \& Sampson, J. R. Encoding and arousal factors in free recall of verbal and visual material. Psychonomic Science, 1968, 12, 73-74.

Murdock, B. B., \& Carey, S. T. Release from interference in single-trial free recall. Journal of Verbal Learning \& Verbal Behavior, 1972, 11, 398-402.

Murdock, B. B., Jr., \& Walker, K. D. Modality effects in free recall. Journal of Verbal Learning \& Verbal Behavior, 1969, 8, 665-676.

Paivio, A. Mental imagery in associative learning and memory. Psychological Review, 1969, 76, 241-263.

Paivio, A. Imagery and verbal processes. New York: Holt, Rinehart, \& Winston, 1971.

Prytulak, L. S. Natural language mediation. Cognitive Psychology, 1971, 2, 1-56.

Rothkopf, E. Z. Incidental memory for location of information in text. Journal of Verbal Learning \& Verbal Behavior, 1971, $10,608-613$.

Rundus, D. Analysis of rehearsal processes in free recall. Journal of Experimental Psychology, 1971, 89, 63-77.

Sampson, J. R. Free recall of verbal and non-verbal stimuli. Quarterly Journal of Experimental Psychology, 1970, 22, 215-221.

Shiffrin, R. M., \& Atkinson, R. C. Storage and retrieval processes in long-term memory. Psychological Review, 1969, 76, 179-193.

Underwood, B. J. Attributes of memory. Psychological Review, 1969, 76, 559-573.

Wickens, D. D. Encoding categories of words: An empirical approach to meaning. Psychological Review, 1970, 77, 1-15.

Zechmeister, E. B., \& McKillip, J. Memory for place on the page. Paper presented at the 43rd Annual Meeting of the Midwestern Psychological Association, Detroit, May 1971.

(Received for publication December 27, 1972; revision received March 23,1973 .) 